\title{
Superconductivity, Josephson coupling and order parameter symmetry in striped cuprates
}

\author{
A. H. Castro Neto ${ }^{1}$ and F. Guinea ${ }^{2}$ \\ 1 Department of Physics, University of California, Riverside, CA, 92521 \\ ${ }^{2}$ Instituto de Ciencia de Materiales, Consejo Superior de Investigaciones Científicas, Cantoblanco, E-28049, Madrid, Spain
}

(January 2, 2018)

\begin{abstract}
We consider a renormalization group study of the problem of coupled stripes of holes in cuprates. We use a model of a mesh of horizontal and vertical stripes and study the problem of superconductivity via the Josephson coupling. We discuss the evolution of the Fermi surface with doping and temperature, the existence of Luttinger and/or Fermi liquid behavior, the presence of pre-formed Cooper pairs and the symmetry and magnitude of the superconducting order parameter.
\end{abstract}

PACS numbers:74.20.Mn, 74.50.+r, 74.72.Dn, 74.80.Bj

After more than ten or so years after their discovery, there is still a debate about the microscopic physics of high temperature superconductors. Due to strong electronic correlations transition metal oxides show a rich variety of phenomena such as anomalous transport, incommensurate magnetic fluctuations and phase separation. Incommensurate magnetic effects have been observed in $\mathrm{La}_{2-x} \mathrm{Sr}_{x} \mathrm{CuO}_{4}$ [1] and have been shown to have their origin in the formation of striped phases in related compounds such as $\mathrm{La}_{1.6-x} \mathrm{Nd}_{0.4} \mathrm{Sr}_{x} \mathrm{CuO}_{4}$ [2] and antiferromagnetic insulators such as $\mathrm{La}_{2-x} \mathrm{Sr}_{x} \mathrm{NiO}_{4+y}$ [3]. Recently it has been reported that the same incommensurate effects are observed in $\mathrm{YBa}_{2} \mathrm{Cu}_{3} \mathrm{O}_{7-\delta}$ and $\mathrm{Bi}_{2} \mathrm{Sr}_{2} \mathrm{CaCu}_{2} \mathrm{O}_{8-x}$ [4]. Furthermore, various different experiments point towards a low dimensional picture of these compounds and to a closeness to quantum critical behavior which can be interpreted in terms of static stripe formation [5]. In the stripe picture the low dimensionality is due to charge segregation into domain walls [6]. Incommensurability appears because of the magnetic domain formation. In the antiferromagnetic phase stripes seem to explain well the available data [7]. In this paper we study the problem of superconductivity and Fermi surface evolution with doping and temperature in a model proposed recently in which the stripes form a mesh of horizontal and vertical Luttinger liquids that hybridize with each other [8]. A similar model was proposed sometime ago in order to study the crossover from repulsively interacting one-dimensional Luttinger liquids to two-dimensional Fermi liquids [9] and has similarities with models of c-axis tunneling 10 .

Our analysis is based on renormalization group calculations [11] and we show that the model not only provides a very simple picture of the Fermi surface in underdoped cuprates but also agrees with recent experiments, especially angle resolved photo-emission (ARPES) 12 11. We also calculate the relevant energy scales for the model and propose new experiments that can test our ideas. Our model differs substantially from earlier work on the effects of stripes in ARPES [15].

The model consists of horizontal and vertical stripes in different $\mathrm{CuO}_{2}$ planes described as Luttinger liquids with a characteristic interaction parameter $g$ where $g>1$ $(g<1)$ for attractive (repulsive) interactions [16] and separated from each other by antiferromagnetic regions. The Hamiltonian of the problem is written as (we use units such that $\hbar=k_{B}=1$ )

$$
\mathcal{H}=\sum \mathcal{H}_{x, n_{y}}+\sum \mathcal{H}_{y, n_{x}}+\mathcal{H}_{T}
$$

where $\mathcal{H}_{x n_{y}}$ describes a $1 \mathrm{D}$ chain with lattice spacing $a$ in the $x$ direction, which intersects the $y$-axis at $n_{y} \ell$ where $n_{y}$ is an integer and $\ell=N a$ is the inter-stripe distance. A similar notation is used to define $\mathcal{H}_{y, n_{x}} . \mathcal{H}_{T}$ describes tunneling between vertical and horizontal chains with amplitude $t$. We assume that the coupling is weak and treat $\mathcal{H}_{T}$ as a perturbation on the Luttinger liquid. We can define an extended Brillouin zone, in terms of the lattice constant of each individual chain, $a$. Each set of chains gives rise to a band in the extended zone. In the absence of hybridization the electrons are localized on the stripes and the Fermi surface is shown in Fig.1(a) where the horizontal and vertical bands are bounded by the lines at $\pm k_{F}$ where $k_{F}=\pi n / 2$ is the Fermi momentum for a chain with linear density $n$.

The scaling equations for the coupling constants are obtained by tracing out high energy degrees and rescaling towards low energies [11]. The scaling equation for the tunneling is given by

$$
\partial_{l} \tilde{t}=\tilde{c}_{4} \tilde{t}
$$

where $\tilde{c}_{4}=1-1 / \tilde{g}$ where $1 / \tilde{g}=(g+1 / g) / 4$ and $\tilde{t}$ is the ratio between the hopping in $\mathcal{H}_{T}$ and the infrared cutoff, $\Lambda(d l=d|\Lambda| / \Lambda)$ 17. The renormalized tunneling energy is $t_{R}=t\left(t / \omega_{c}\right)^{\left(1-c_{4}\right) / \tilde{c}_{4}}(a / \ell)^{1 / \tilde{c}_{4}}$ where $\omega_{c}$ a bandwidth cut-off [9]. In the absence of tunneling, the electrons have a well defined momentum in the direction parallel to the stripe where they are located. $\mathcal{H}_{T}$ induces the folding of the Brillouin zone shown in Fig. 1) (a) into $N \times N$ smaller zones. However, these are higher order effects in terms of the tunneling and distortions of the mesh, induced either by static disorder or thermal fluctuations [18, will smear these additional "shadow" bands [19]. Inter-chain tunneling will also hybridize the states derived from horizontal and vertical bands. Observe that for a doping amount of 
$x$ holes there are $1 / N-x$ electrons participating on this Fermi surface. The other $1-1 / N$ electrons form the surrouding antiferromagnetic background. Since the antiferromagnetic and stripe electrons are in equilibrium they must have the same chemical potential (so that the lower Hubbard band of the antiferromagnet is pinned at the stripe Fermi surface). Thus, superimposed to the Fermi surface of Fig.11(a), one must have a diamond shaped, and somewhat blurred, occupation in momentum space for the antiferromagnetic electrons, with area proportional to $1-1 / N$. The final result is shown on Fig.11(b). The total area seen by ARPES should be proportional to $1-x$, corresponding to a large Fermi surface. These results agree well with the available ARPES data [12,13].

(a)

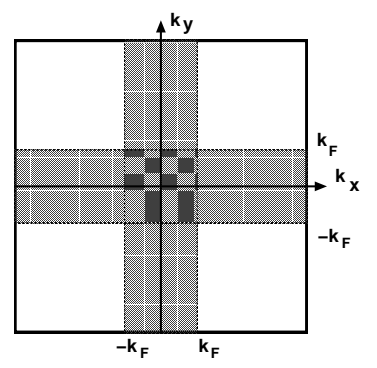

(b)

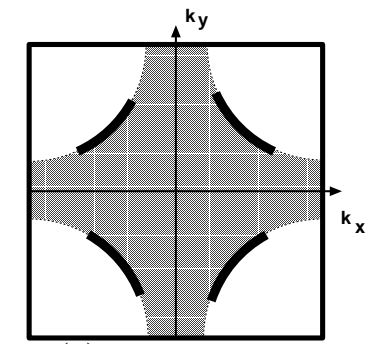

FIG. 1. (a) Fermi surface for the stripes without hybridization; (b) Fermi surface with hybridization: the continuous line shows the region of Fermi liquid behavior (finite residue) and the doted line shows region of non-Fermi liquid behavior.

In most points of the Brillouin zone, the horizontal and the vertical bands are not degenerate and there is a splitting $\delta_{k_{x}, k_{y}}$ between bands. At zero temperature $\delta_{k_{x}, k_{y}}$ cuts off the RG flow. If $\delta_{k_{x}, k_{y}}>t_{R}$ the vertical and horizontal stripes decouple and coherence effects are not expected. As shown in ref. [9] the quasiparticle residue vanishes in the regions close to $( \pm \pi / a, 0)$ and $(0, \pm \pi / a)$ and the system behaves like a Luttinger liquid. The only exception are the points close $\left( \pm k_{F}, \pm k_{F}\right)$ where $\delta_{ \pm k_{F}, \pm k_{F}}=0$. When $\delta_{k_{x}, k_{y}}<t_{R}$ the horizontal and vertical bands will become strongly hybridized, and a quasiparticle residue is finite [20] leading to local Fermi liquid behavior. The crossover from Luttinger to Fermi liquid along the Fermi surface is defined by $t_{R} \approx \delta_{k_{x}, k_{y}}$ as shown in Fig. Il(b). It turns out however that the regions of non-Fermi liquid behavior are nested and therefore will be subjected to instabilities [21]. An interesting consequence of this scenario is that there is a crossover from Luttinger liquid to Fermi liquid behavior also as a function of $\ell$ as $\ell$ decreases. There is a minimum value of $\ell$, say $\ell^{*}$, for which $\delta\left(\ell^{*}\right) \approx t_{R}\left(\ell^{*}\right)$. Since $\ell$ is a monontonic decreasing function of $x$ [8] there is a crossover doping $x^{*}$ such that for $x<x^{*}\left(x>x^{*}\right)$ we have Luttinger (Fermi) liquid behavior.

Interaction processes do not need to involve an energy cost, even if they conserve crystal momentum. We can use a standard diagrammatic analysis to study the interactions which can be generated by the combined effect of the intra-chain couplings and the inter-chain hopping. The irreducible diagrams that can be built starting with two electrons in either the horizontal or the vertical chains, which interact between them, hop into the other set of chains, and interact again. Besides the usual pairing process within each chain which is shown in Fig 2(a) there are two types of possible couplings which satisfy momentum conservation which are depicted in Fig.2(b) : i) The Cooper pair channel: Cooper pair in the horizontal chains is transferred to the vertical chains, or vice-versa; ii) The transfer of Cooper pairs with total momentum $\left( \pm 2 k_{F}, \pm 2 k_{F}\right)$. There are also direct (Fig.2(c)) and exchange (Fig.2(d)) diagrams which give a vanishing contribution ibecause of phase space restrictions [11]. These processes can all be written as $\hat{A}_{v h}=\hat{O}_{v}^{\dagger} \hat{O}_{h}$, where $\hat{O}$ is an operator which creates, or destroys, a pair of electrons in one chain. As each separated chain is a Luttinger liquid, the $\hat{O}$ 's acquire anomalous dimensions relative to the non-interacting fixed point. The successive application of these operators generate interactions which take a pair of electrons from one set of chains into the other set and back again. Thus, upon scaling towards low energies, interactions of the type $\hat{A}_{h h}$ and $\hat{A}_{v v}$ will also be generated. In general, the operator $\hat{A}$ has to be considered a $2 \times 2$ matrix. It can be shown [22] that the $\hat{O}$ associated with processes $i i$ ) is less relevant than the operator which describes processes of type $i$ ). Note that, as our scheme implies a summation to all orders of intra-chain effects, the propagators shown in Fig.2 acquire anomalous dimensions [16]. The scaling equation for the matrix $\hat{A}_{i j}$ has the Riccati form:

$$
\partial_{l} \hat{A}=-\tilde{c}_{1}(g, l) \tilde{t}^{2} \sigma_{x}+2 \tilde{c}_{2}(g) \hat{A}-\tilde{c}_{3}(g, l) \hat{A}^{2}
$$

where $\sigma_{x}$ is a Pauli matrix. $\tilde{c}_{1}(g, l)=c_{1} e^{4(g-1) l}$ represents the generation of the Cooper pair tunneling starting from the tunneling of individual electrons. In a conventional junction between superconductors, this is the Josephson coupling $\left(c_{1}>0\right.$ unless the tunneling takes place through a magnetic region [23]). The second term is the scaling dimension of the operators with $\tilde{c}_{2}>0$ $\left(\tilde{c}_{2}<0\right)$ for attractive (repulsive) intra-chain interactions. $\tilde{c}_{3}(g, l)=e^{2(g-1) l}$ is associated with the twodimensional pairing on the Fermi surface in Fig. 11(a) [11]. Observe that due to symmetry $\hat{A}=A I+B \sigma_{x}$ where $A$ and $B$ are real numbers and $I$ is the identity matrix. It is clear from equation (11) that it is useful to work with the eigenvalues of $\hat{A}$ :

$$
\tilde{\lambda}_{ \pm}=A \pm B
$$


After a redefinition of the variables $\left(\lambda_{ \pm}=e^{2(g-1) l} \tilde{\lambda}_{ \pm}\right.$, $\left.\tau=e^{3(g-1) l} \tilde{t}\right)$ the $\mathrm{RG}$ equations read:

$$
\begin{aligned}
\partial_{l} \lambda_{+} & =-c_{1} \tau^{2}+2 c_{2}(g) \lambda_{+}-\lambda_{+}^{2} \\
\partial_{l} \lambda_{-} & =c_{1} \tau^{2}+2 c_{2}(g) \lambda_{-}-\lambda_{-}^{2} \\
\partial_{l} \tau & =c_{4}(g) \tau
\end{aligned}
$$

where $c_{2}(g)=g-1+\tilde{c}_{2}$ and $c_{4}(g)=3(g-1)+\tilde{c}_{4}(g)$. The RG flow has four fixed points: $(0,0,0),\left(2 c_{2}, 0,0\right)$, $\left(0,2 c_{2}, 0\right)$ and $\left(2 c_{2}, 2 c_{2}, 0\right)$. The $(0,0,0)$ is the noninteracting fixed point; $\left(2 c_{2}, 2 c_{2}, 0\right)$ is the non-interacting fixed point of isolated chains but with superconducting fluctuations. The physics of the other two fixed points is more complex. To gain more insight we study the RG flow when $\tau=0$.

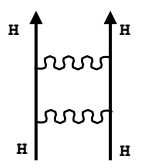

(a)

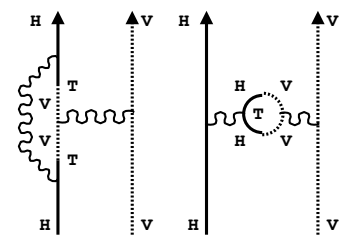

(c)
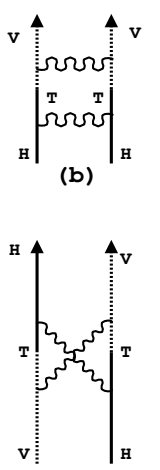

(d)
FIG. 2. (a) Cooper pair diagram within a horizontal stripe (H); (b) Cooper pair diagram for a pair in a horizontal stripe (H) tunneling $(\mathrm{T})$ to a vertical stripe $(\mathrm{V})$; (c) direct processes; (d) exchange processes.

For attractive interactions the flow is shown in Fig. 3(a). Observe that $\left(2 c_{2}, 2 c_{2}, 0\right)$ is stable whereas the non-interacting fixed point is unstable; the other two fixed points are also unstable. $\left(2 c_{2}, 0,0\right)$ is unstable in the negative $\lambda_{+}$direction and from the diagram of Fig.2( (b) we see that the instabilities is of the superconducting type. One can understand the strong coupling fixed point in a very simple way: observe that $\lambda_{-}$is constant and $\lambda_{+}<<-1$. From (2) we have $A<B<<-1$. It implies a superconducting instability in which the sign of the order parameter is the same along horizontal and vertical stripes; this is equivalent to $s$-wave pairing. $\left(0,2 c_{2}, 0\right)$ is unstable in the negative $\lambda_{-}$direction while $\lambda_{+}$is constant which implies $A<<-1$ and $B>>1$. The coefficients have opposite sign which means that the sign of the order parameter in the vertical stripes is opposite to the sign in the horizontal stripes leading to what is called a $\pi$-junction [23]. Since the stripes are oriented along the crystallographic directions this is equivalent to $d$-wave pairing. For repulsive interactions the s-wave and d-wave pairing persist and the flow is shown on Fig. 3(b). If $\tau \neq 0$ the hopping is relevant (except for strongly attractive interactions). If $c_{1}>0$ the system flows towards $\mathrm{s}$-wave and if $c_{1}<0$ the flow is reversed towards d-wave superconductivity. One also expects that in the presence of repulsive interactions the mesh should be insulating because of the intrinsic disorder in the $\mathrm{CuO}_{2}$ planes [24]. A mesh like the one proposed here is very sensitive to disorder and impurities, such as Zn. Note that Zn reduces drastically the superfluid density [25]. The attraction within each stripe is provided by the surrounding antiferromagnet [6,26]. The model proposed here should also be useful in describing artificial Josephson arrays 27.

Since the flow is divergent at some finite scale $l^{*}$ we estimate the gap from the scaling equations by studying the flow close to the singularity. At this point the tunneling amplitude is constant and given by $\tau_{*}=\tau\left(l^{*}\right)$. If $\tau_{*}^{2}>c_{2} / c_{1}$ the gap has a BCS form,

$$
\Delta=\omega_{p} \exp \left\{-\frac{\frac{\pi}{2}-\arctan \left(\frac{\left|\lambda_{0}-c_{2}\right|}{\sqrt{c_{1} \tau_{*}^{2}-c_{2}^{2}}}\right)}{\sqrt{c_{1} \tau_{*}^{2}-c_{2}^{2}}}\right\}
$$

where $\lambda_{0}$ is the coupling constant and $\omega_{p}$ is the characteristic pairing energy. In this limit one expects usual BCS behavior with well defined quasiparticles in the superconducting state. Eq. (4) reduces to the usual BSC gap $\left(\Delta_{B C S}=\omega_{p} e^{\left.-1 /\left|\lambda_{0}\right|\right)}\right)$ in the non-interacting limit $\left(c_{1,2} \rightarrow 0\right)$. In the opposite limit, $\tau_{*}^{2}<c_{2} / c_{1}$, Luttinger liquid behavior is obtained with a non-BCS gap given by

$$
\Delta=\omega_{p}\left[\frac{\lambda_{0}-\sqrt{c_{2}^{2}-c_{1} \tau_{*}^{2}}-c_{2}}{\lambda_{0}+\sqrt{c_{2}^{2}-c_{1} \tau_{*}^{2}}-c_{2}}\right]^{-\left(2 \sqrt{c_{2}^{2}-c_{1} \tau_{*}^{2}}\right)^{-1}}
$$

which, when $c_{1} \rightarrow 0$, has the interesting form $\Delta_{L}=$ $\omega_{p}\left(1+c_{2} /\left|\lambda_{0}\right|\right)^{-1 / c_{2}}$ which raises much faster with the coupling constant than the usual BCS result.

Let us consider the tunneling of Cooper pairs between transverse stripes $A$ and $B$ through the antiferromagnetic regions. Since the pair is in a singlet state the tunneling process occurs in such a way that a given spin order the sign of the wavefunction is reversed [23]. This is the so-called a $\pi$-junction and implies that $c_{1}<0$ in the RG flow. The argument is valid if the tunneling occurs through a region smaller than the magnetic correlation length, $\xi_{M}$ [28]. A similar mechanism for d-wave pairing involving tunneling through a finite antiferromagnetic region was found by Bonesteel 29. Assuming that $c_{1} \leq 0$ the tunneling through the antiferromagnet only takes place if $\xi_{M}\left(T^{*}\right) \approx a$. Thus, there is a crossover temperature $T^{*}$ at above which $c_{1}=0$ [30]. $T^{*}$ can be roughly estimated assuming that the antiferromagnet is in the quantum critical regime [28] where $T^{*} \approx c / a(c$ is the spin wave velocity). 


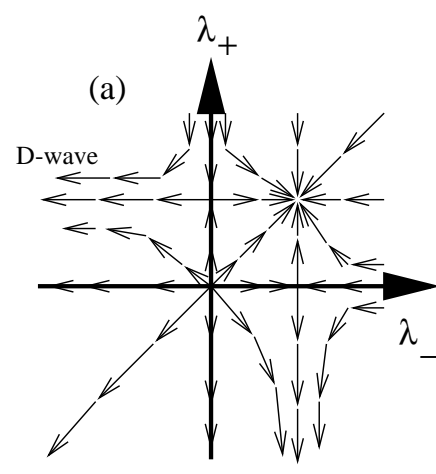

S-wave
S-wave

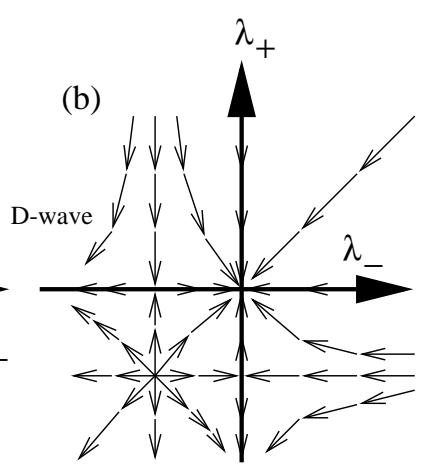

FIG. 3. Renormalization group flow in the $\tau=0$ plane: (a) attractive interactions; (b) repulsive interactions.

In conclusion, besides the shape of the Fermi surface, the existence of "shadow" bands, the co-existence of Fermi liquid and non-Fermi liquid behavior, the possibility of non-BCS and BCS behavior and s- and/or dwave pairing we have three different temperature scales in this problem, namely, $T^{*}, \delta$ and $\Delta$. Consider first the case such that $T^{*}>\delta>\Delta$. If $T^{*}>T>\delta$ the pairing takes place but there is no coherence between the stripes. There are pre-formed pairs and a gap to spin excitations due to the formation of singlets. This effect is observed experimentally in infrared absorption and nuclear magnetic resonance (NMR) [31]. If $\delta>T$ tunneling takes place and a gap opens at the $( \pm \pi / a, 0)$, $(0, \pm \pi / a)$ regions of the Fermi surface but no phase coherence is observed. This case has similarities to a recent model proposed by Geshkenbein et al. for the physics of the Fermi surface with pre-formed pairs 32]. In the other scenario, $\delta>T^{*}>\Delta$, the physics is quite different. For $\delta>T>T^{*}$ a Luttinger liquid is formed close to $( \pm \pi / a, 0),(0, \pm \pi / a)$ but no Cooper pairs are found. When $T^{*}>T$ the Luttinger liquid regions of the Fermi surface become gapped due to pair formation. At lower temperatures superconductivity is obtained. While the first case is closer to a conventional superconducting instability the last one shows direct transition from a Luttinger liquid to a superconductor [10]. This picture agrees with the available data in the underdoped cuprates [12,13 and provides a microscopic origin for the boson modes proposed by Shen and Schrieffer 14 in terms of collective modes of the Luttinger liquid. Observe that in the underdoped compounds we predict weak dependence of $k_{F}$ on doping [28].

Finally, we can predict a new effect as we change the ratio $r \approx T^{*} / \delta \approx\left(c / v_{F}\right)(\ell / a)$. Close to the metal-insulator transition one expects $r>>1$ because $c$ can be large and $\ell / a \approx 1 / x>>1$. In this case the first scenario applies and as the system is cooled down a spin gap must be observed before a gap opens at $( \pm \pi / a, 0),(0, \pm \pi / a)$ in the Fermi surface. At larger doping (but smaller than $x^{*}$ ), that is, when $r<<1$, one expects that no gap will appear in the Fermi surface (because of Luttinger behav- ior) and at lower temperatures a spin gap appears before the system superconducts.

We thank G. Aeppli, L. Balents, D. Baeriswyl, G. Castilla, A. Chubukov, M. P. A. Fisher, E. Fradkin, N. Hasselmann, S. Kivelson, C. Nayak and S. White for useful discussions, and the Benasque Center for Physics, for its hospitality. A. H. C. N. is an Alfred P. Sloan fellow. F. G. is thankful to CICyT (Spain) for financial support (grant PB96-0875).

Note: Since completing this work, we became aware of numerical work on stripes in the t-J model [33] which seems to be consistent with our results.

[1] S. W. Cheong et al., Phys. Rev. Lett. 67, 1791 (1991).

[2] J. M. Tranquada et al., Nature, 375, 561, (1995).

[3] J. M. Tranquada et al., Phys. Rev. Lett. 73, 1003 (1994).

[4] P. Dai et al., cond-mat 9707112.

[5] T. E. Mason et al., Phys. Rev. Lett. 77, 1604 (1996); G. Aeppli et al., cond-mat 9801169.

[6] V. Emery and S. Kivelson, Physica C 209, 597 (1993); V. Emery et al., cond-mat 9610094.

[7] A. H. Castro Neto and D. Hone, Phys. Rev. Lett. 76, 2165 (1996), cond-mat 9701042.

[8] A. H. Castro Neto, Phys. Rev. Lett. 78, 3931 (1997).

[9] F. Guinea and G. Zimanyi, Phys.Rev.B 47, 501 (1993).

[10] S. Chakravarty and P. W. Anderson, Phys. Rev. Lett. 72, 3859 (1994).

[11] R. Shankar, Rev. Mod. Phys. 66, 129 (1994).

[12] D. S. Marshall et al., Phys. Rev. Lett. 76, 4841 (1996).

[13] M. Randeria and J. C. Campuzano, cond-mat 9709107.

[14] Z. -X. Shen and J. R. Schrieffer, Phys. Rev. Lett. 78, 1771 (1997).

[15] M. I. Salkola et al., Phys. Rev. Lett. 77, 155 (1996).

[16] J. Voit, Rep. Prog. Phys. 58, 977 (1995).

[17] H. -H. Lin et al., cond-mat 9703055.

[18] H. Eskes et al., Phys. Rev. B 54, 724 (1996).

[19] P. Aebi et al., Phys. Rev. Lett. 72, 2757 (1994).

[20] X. -G. Wen, Phys. Rev. B 42, 6623 (1990).

[21] D. Pines, Z. Phys. B 103, 129 (1997).

[22] J. M. P. Carmelo et al., Phys. Rev. B 55, 7565 (1997).

[23] L. N. Bulaeveskii et al., JETP Lett. 25, 290 (1977); B. Spivak and S. Kivelson, Phys. Rev. B 43, 3740 (1991).

[24] G. S. Boebinger et al., Phys. Rev. Lett. 77, 5417 (1996).

[25] A. H. Castro Neto and A. V. Balatsky, unpublished.

[26] G. Castilla and A. H. Castro Neto, unpublished.

[27] H. R. Shea and M. Tinkham, Phys. Rev. Lett. 79, 2324 (1997); P. Chandra et al., Phys. Rev. Lett. 75, 713 (1995).

[28] A. H. Castro Neto, Z. Phys. B, 103, 185 (1997).

[29] N. E. Bonesteel, unpublished.

[30] S. .M. Hayden et al., Phys. Rev. Lett. 76, 1344 (1996).

[31] C. Berthier et al., J. Physique I 6, 2205 (1996); A. Puchkov et al., J. Phys.-Cond. Matter 8, 10049 (1996).

[32] V. B. Geshkenbein et al., Phys. Rev. B 55, 3173 (1997).

[33] S. White and D. J. Scalapino, cond-mat/9801274. 